\title{
Determining the role of natural SARS-CoV-2 infection in the death of domestic pets: I0 cases (2020-202I)
}

\author{
Ann Carpenter DVM, MPH \\ Ria R. Ghai $\mathrm{PhD}$ \\ Joy Gary DVM, PhD \\ Jana M. Ritter DVM \\ Francisco R. Carvallo DVM, DSC \\ Diego G. Diel DVM, PhD \\ Mathias Martins DVM, $\mathrm{PhD}$ \\ Julia Murphy DVM \\ Betsy Schroeder DVM, PhD \\ Kevin Brightbill DVM \\ Deepanker Tewari BVSc, $\mathrm{PhD}$ \\ Lore Boger VMD, MS \\ Julie Gabel DVM, MPH \\ Robert Cobb DVM \\ Janemarie Hennebelle DVM, MPVM \\ James B. Stanton DVM, PhD \\ Kathryn McCullough DVM \\ Yung-Yi C. Mosley BVM, PhD \\ Hemant K. Naikare BVsc, $\mathrm{PhD}$ \\ Rachel Radcliffe DVM, MPH \\ Boyd Parr DVM \\ Gary Balsamo DVM, MPH \\ Brent Robbins DVM \\ David Smith DVM \\ Sally Slavinski DVM, MPH \\ Carl Williams DVM \\ Doug Meckes DVM \\ Dee Jones DVM \\ Tony Frazier DVM \\ Kelley Steury DVM, MPH \\ Jane Rooney DVM \\ Mia Torchetti DVM, PhD \\ Natalie Wendling DVM, MPH \\ Dustin Currie PhD, MPH \\ Casey Barton Behravesh DVM, DrPH \\ Ryan M. Wallace DVM, MPH
}

OBJECTIVE

To establish a pathoepidemiological model to evaluate the role of SARSCoV-2 infection in the first 10 companion animals that died while infected with SARS-CoV-2 in the US.

\section{ANIMALS}

10 cats and dogs that tested positive for SARS-CoV-2 and died or were euthanized in the US between March 2020 and January 202I.

\section{PROCEDURES}

A standardized algorithm was developed to direct case investigations, determine the necessity of certain diagnostic procedures, and evaluate the role, if any, that SARS-CoV-2 infection played in the animals' course of disease and death. Using clinical and diagnostic information collected by state animal health officials, state public health veterinarians, and other state and local partners, this algorithm was applied to each animal case.

\section{RESULTS}

SARS-CoV-2 was an incidental finding in 8 animals, was suspected to have contributed to the severity of clinical signs leading to euthanasia in I dog, and was the primary reason for death for I cat.

\section{CONCLUSIONS AND CLINICAL RELEVANCE}

This report provides the global community with a standardized process for directing case investigations, determining the necessity of certain diagnostic procedures, and determining the clinical significance of SARS-CoV-2 infections in animals with fatal outcomes and provides evidence that SARSCoV-2 can, in rare circumstances, cause or contribute to death in pets.

ARS-CoV-2, the virus that causes COVID-19, is be-
lieved to have originated in horseshoe bats and
emerged in the human population in late 2019 , pos-
sibly through an intermediate host. ${ }^{1-3}$ Viral spillover
from people into animal populations has occurred
naturally throughout the globe in farmed mink, zoo
animals including big cats and great apes, and com-
panion animals. ${ }^{4,5}$ Along with farmed mink, compan-
ion animals (ie, dogs and cats) are the most common-
ly infected animal group and are primarily infected
by owners with COVID-19. Nearly all companion ani-
mals infected with SARS-CoV-2 have had subclinical
infection or have developed mild illness. ${ }^{6-9}$
Severe illness due to SARS-CoV-2 infection has not
been established in companion animals. Five compan-
ion animals positive for SARS-CoV-2 outside the US are
known to have died: a dog in the Netherlands, 2 dogs

From the CDC, Atlanta, GA 30333 (Carpenter, Ghai, Gary, Ritter, Wendling, Currie, Behravesh, Wallace); Department of Biomedical Sciences \& Pathobiology, Virginia-Maryland College of Veterinary Medicine, Virginia Tech, Blacksburg, VA 24060 (Carvallo); Department of Population Medicine and Diagnostic Sciences, Animal Health Diagnostic Center, College of Veterinary Medicine, Cornell University, Ithaca, NY I4853 (Diel, Martins); Virginia Department of Health, Richmond, VA 23219 (Murphy); Pennsylvania Bureau of Epidemiology, Pennsylvania Department of Health, Harrisburg, PA I7I20 (Schroeder); Pennsylvania Bureau of Animal Health and Diagnostic Services, Harrisburg, PA I7II0 (Brightbill, Tewari, Boger); Georgia Department of Public Health, Atlanta, GA 30303 (Gabel); Georgia Department of Agriculture, Atlanta, GA 30334 (Cobb, Hennebelle); Department of Pathology, University of Georgia College of Veterinary Medicine, Athens, GA 30602 (Stanton, McCullough); Tifton Veterinary Diagnostic \& Investigational Laboratory (TVDIL), Tifton, GA 3I793 (Mosley, Naikare); South Carolina Department of Health \& Environmental Control, Columbia, SC 2920I (Radcliffe); Livestock Poultry Health, Clemson University, Columbia, SC 29224 (Parr); Infectious Disease Epidemiology Section, Office of Public Health, Louisiana Department of Health, New Orleans, LA 70160 (Balsamo); Louisiana Department of Agriculture and Forestry, Baton Rouge, LA 70806 (Robbins); Division of Animal Industry, New York State Department of Agriculture and Markets, Albany, NY 12235 (Smith); Bureau of Communicable Disease, New York City Department of Health and Mental Hygiene, Queens, NY IIIOI (Slavinski); North Carolina Division of Public Health, Raleigh, NC 27699 (Williams); North Carolina Department of Agriculture and Consumer Services, Raleigh, NC 2760I (Meckes); Alabama Department of Public Health, Montgomery, AL 36130 (Jones); Alabama Department of Agriculture and Industries, Montgomery, AL 36107 (Frazier); Thompson Bishop Sparks State Diagnostic Laboratory, Auburn, AL 36832 (Steury); Veterinary Services Strategy and Policy, APHIS, USDA, Ft Collins, CO 80526 (Rooney); National Veterinary Services Laboratories, Veterinary Services Diagnostics and Biologics, APHIS, USDA, Ames, IA 50010 (Torchetti). Dr. Gary's present address is StageBio, 5930 Main St, Mount Jackson, VA 22842.

Address correspondence to Dr. Carpenter (pzy4@cdc.gov). 
in Germany, a cat in Spain, ${ }^{5}$ and a cat in the UK. ${ }^{10}$ The role of SARS-CoV-2 in the dog deaths remains unknown. Postmortem investigation of the cat in Spain implicated a preexisting cardiac condition as the cause of death. ${ }^{11}$

In the US, approximately 3,625 animals were tested between March 2020 and January 2021. Of these, 94 of these companion animals (cats and dogs) met the USDA national case definition for SARS-CoV-2 in animals and were therefore confirmed positive for SARS-CoV-2. ${ }^{12,13}$ While most of these animals were subclinical or mildly ill, 10 (10.6\%) companion animals died or were euthanized around the time of SARS-CoV-2 diagnosis. The purpose of this case series is to examine the characteristics of these companion animals and determine whether their deaths were attributable to SARS-CoV-2.

\section{Materials and Methods}

The CDC and USDA recommend diagnostic testing for companion animals when: 1) the animal has an epidemiological link to a person or animal with SARSCoV-2 or to a high-risk environment and 2) the animal has clinical signs consistent with SARS-CoV-2 ${ }^{14}$. Clinical signs consistent with SARS-CoV-2 infection include coughing, sneezing, nasal or ocular discharge, difficulty breathing, shortness of breath, vomiting, diarrhea, lethargy, or fever. The decision to test companion animals for SARS-CoV-2 should be made using a one-health approach and involve owners, veterinarians, and state animal and public health agencies. ${ }^{14}$ Presumptive positive SARS-CoV-2 results are reported to the submitting veterinarian and state health officials and may be subject to confirmatory testing at the USDA National Veterinary Services Laboratories (NVSL). ${ }^{15,16}$ Although investigation details of animal SARS-CoV-2 infections are not notifiable to federal agencies, the CDC provided state agencies with standardized data collection tools for epidemiological investigations to characterize animal history, clinical signs and outcomes, and transmission dynamics between people and animals. ${ }^{17}$ State agencies provided SARS-CoV-2 case details to federal agencies voluntarily.

\section{Case selection criteria}

All companion animals included in this case series had exposure to a person with COVID-19 and new or worsening illness that resulted in submission of diagnostic testing for SARS-CoV-2 by their veterinarian, and all these animals died or were euthanized. This report spans the time period of March 2020 to January 2021. During this time, 10 companion animals were identified to fit these criteria.

\section{Medical records review}

Cases were reviewed by federal and state health officials, attending veterinarians, and, where applicable, pathologists. Where possible, tissue samples were submitted to local pathologists or pathologists in the CDC's Infectious Diseases Pathology Branch (IDPB) for further evaluation, including histology, immunohistochemistry (IHC), real-time PCR (RT-PCR), or in situ hybridization for SARS-CoV-2 and other pathogens. ${ }^{18}$ An algorithm was developed to help describe the case investigation process and determine the role of SARS-CoV-2 in these cases (Figure I). Four factors were considered: clinical signs consistent with SARS-

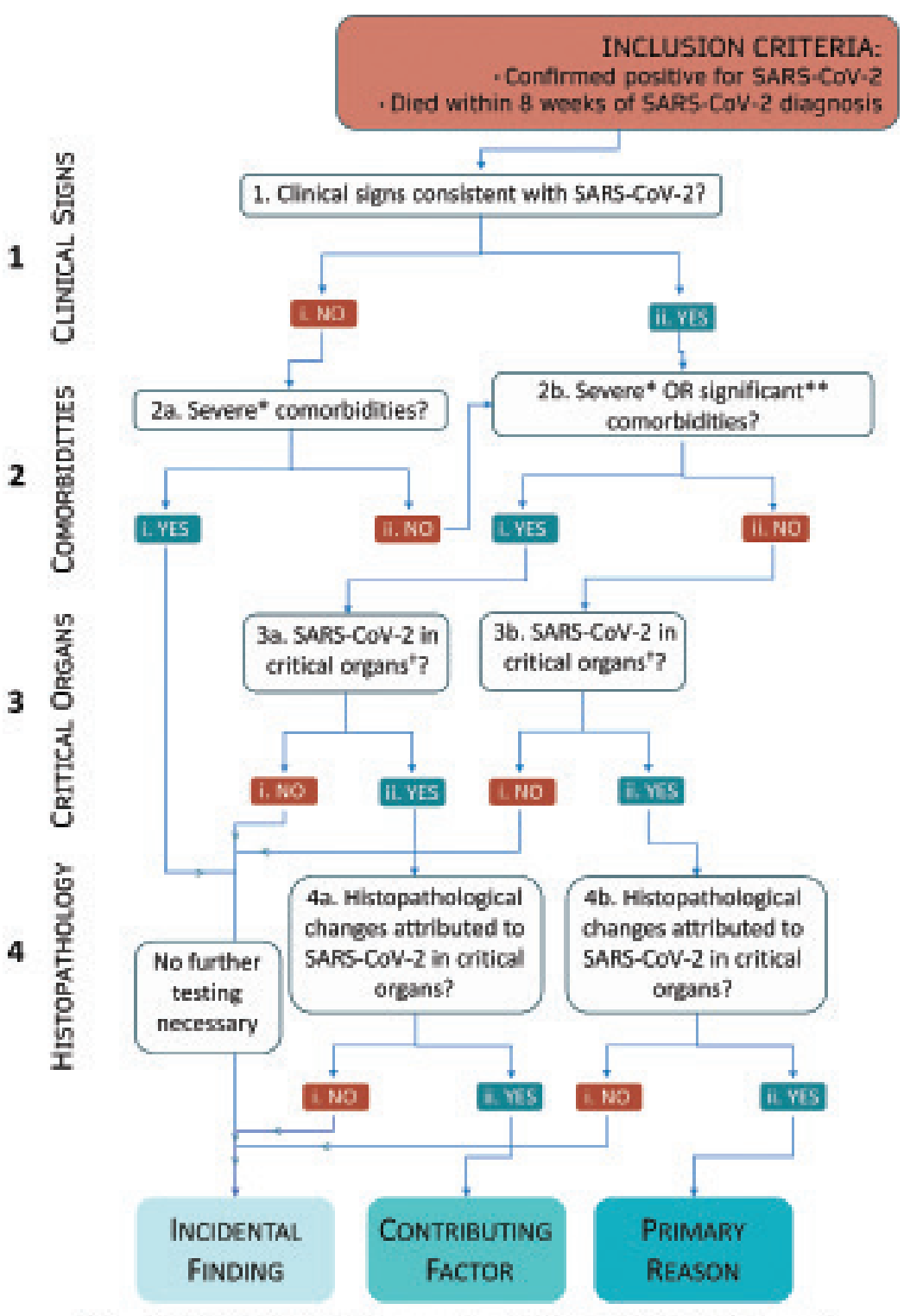

Role of SARS-CoV-2 in Companion Animal Death/Euthanasia

Figure I-Algorithm used to determine the contribution of SARS-CoV-2 in the death of dogs and cats. *Severe comorbidities are likely to have resulted in death or euthanasia. **Significant comorbidities could contribute to the death of the animal but are unlikely to result in death. †Critical organs include heart, lungs, and brain. 
CoV-2 infection, comorbidities, SARS-CoV-2 antigen or nucleic acid detection in critical organs (ie, lungs, brain, and heart), and evidence of histopathological changes attributed to SARS-CoV-2. SARS-CoV-2 was considered to be the primary cause of death if the animal had consistent clinical signs; absence of another, more likely, cause of death; and viral detection in critical tissues with associated histopathological changes. SARS-CoV-2 was considered to be a contributing factor if comorbidities were present and virus was detected in critical tissues with associated histopathological changes. If there was no evidence of virus in critical tissues and no histopathological changes attributed to SARS-CoV-2 in critical tissues, then the infection was considered incidental. In cases where no necropsy or further tissue evaluation was performed, veterinarians and veterinary pathologists used the available clinical history and antemortem diagnostic results to determine the cause of death.

\section{Results}

The developed standardized algorithm (Figure 1) was applied to each case. All animals met the USDA's confirmed positive case definition for SARS-CoV-2 in animals. ${ }^{19}$ Reported cycle threshold $(\mathrm{Ct})$ values were obtained by RT-PCR using the CDC's N target assay. ${ }^{20}$

\section{Case I}

On April 28, 2020, a dog owner sought veterinary care for a 7-year-old male German Shepherd Dog with signs of lethargy, labored breathing, decreased appetite, and a $13.6-\mathrm{kg}$ weight loss since mid-April. Thoracic radiography revealed an enlarged heart, but ECG results were unremarkable. The dog's owner had been diagnosed with COVID-19 on April 21.

On May 15, and with no improvement, the dog presented to a second veterinarian with tachypnea and severe pallor of the mucous membranes. Blood work revealed severe nonregenerative anemia (RBC count, $1.67 \times 10^{6}$ cells $/ \mu \mathrm{L}$; reference range, 5.39 to $8.70 \times$ $10^{6}$ cells $/ \mu \mathrm{L}$ ) and thrombocytopenia (platelet count, $54 \times 10^{3}$ cells $/ \mu \mathrm{L}$; reference range, 143 to $448 \times 10^{3}$ cells $/ \mu \mathrm{L})$. The WBC count was within reference range at $9.92 \times 10^{3}$ cells $/ \mu \mathrm{L}$ but showed a predominance of monocytes (54.5\%). A screening test for heartworm disease and tick-borne pathogens was negative. Nasal swabs collected on May 15 and 20 were positive for SARS-CoV-2 RNA (RT-PCR; Ct, 31) and negative, respectively. Serum samples collected on May 15 and 20 contained virus-neutralizing antibodies (1:512). Prednisone and doxycycline were administered. The owner reported that the dog had immediate improvement, with increased appetite and energy.

Over the next several weeks, the dog developed urinary incontinence, hematuria, hematemesis, and worsening dyspnea. Blood work on June 21 revealed continued severe nonregenerative anemia and an increasing WBC count ( $72 \times 10^{9}$ cells/L) with a predominance of monocytes (80\%). Large, immature blastic cells, suggestive of a hemic neoplasia, were found on manual differentiation. The dog was euthanized on July 11. A necropsy was not performed. Based on laboratory results and clinical course, the cause of death was consistent with a hemic neoplasia such as acute lymphoid leukemia or lymphoma. This dog had clinical signs attributed to other disease processes (Figure 1, 1.i.) and had severe comorbidities (Figure 1, 2a.i.). Therefore, SARS-CoV-2 infection was concluded to have been an incidental finding (Supplementary Table SI).

\section{Case 2}

On June 19, 2020, a 6-year-old neutered male Boxer mix acutely developed seizures and hypersalivation. Both of its owners had developed symptoms (June 11 and 13) and tested positive for SARS-CoV-2 (June 12 and 15).

The dog was presented to a veterinary clinic on June 20 and was euthanized on June 21 after failing to improve with medical therapy. When it was learned that owners had been diagnosed with COVID-19, a nasal swab for SARS-CoV-2 testing was collected postmortem and confirmed positive by RTPCR (Ct, 27). A necropsy performed on June 24 revealed an intracranial Schwannoma with regionally extensive encephalomalacia and neovascularization involving the optic nerve and overlying brain. Minimal rhinitis and palatitis were also noted; however, the dog did not show respiratory signs. The cause of death was attributed to intracranial Schwannoma and acute secondary hemorrhage. This animal had clinical signs attributed to other disease processes (Figure 1, 1.i.) and had severe comorbidities (Figure 1, 2a.i.). SARS-CoV-2 was determined to be an incidental finding (Supplementary Table S1).

\section{Case 3}

On June 24, 2020, an 11-year-old neutered male domestic shorthair (DSH) cat presented to a veterinary clinic with a 2-day history of dyspnea. No diagnostic testing was performed, no treatments were attempted, and the owner was instructed to return if the clinical signs did not resolve. Between June 7 and June 14, all 4 persons in the household had developed febrile illness and tested positive for COVID-19.

On June 24, the cat's dyspnea worsened and it was taken to an emergency clinic, where clinicians noted tachypnea, hypothermia, and a heart murmur. Thoracic radiography revealed no pneumonia or other abnormalities. Cardiac ultrasonography showed bilateral markedly thickened myocardium and left atrial enlargement, consistent with hypertrophic cardiomyopathy (HCM). While hospitalized on June 25 , the cat remained laterally recumbent with poor mentation. The cat died later that day; necropsy was not performed. After veterinary staff were informed that the animal came from a household that had people with COVID-19, nasal swabs were collected from the cat postmortem and were positive for SARS-CoV-2 by RT-PCR (Ct, 34). A standard respiratory panel was positive for Mycoplasma felis. Since clinical signs and 
cause of death were attributed to HCM, SARS-CoV-2 was concluded to be an incidental finding (Figure 1, 1.i. and 2a.i.; Supplementary Table S1).

\section{Case 4}

On July 13, 2020, a 9-year-old spayed female Boxer mix developed rear limb paresis. Radiographic findings were consistent with vertebral disk disease or spinal neoplasia. At the request of the owner, who had tested positive for SARS-CoV-2 on July 20, a nasal swab was collected from the dog by the veterinarian on July 22 and tested positive for SARS-CoV-2 by RT-PCR (Ct, 30). The dog was euthanized on August 5 due to hind limb plegia, with a presumptive diagnosis of intervertebral disc disease. Necropsy was not performed. A second nasal sample collected by the veterinarian postmortem tested positive by RT-PCR (Ct 37), and serum was positive for virus-neutralizing antibodies. SARS-CoV-2 infection was determined to be an incidental finding, as the dog showed no clinical signs consistent with SARS-CoV-2 (Figure 1, 1.i.) and was euthanized for unrelated reasons (Figure 1, 2a.i.; Supplementary Table S1).

\section{Case 5}

On August 3, 2020, an 8-year-old Newfoundland developed acute respiratory distress and pyrexia (rectal temperature, $40.7^{\circ} \mathrm{C}$ ) and presented to a veterinary clinic. Owners reported that the dog appeared normal that morning. The dog's condition deteriorated over 12 hours, resulting in referral to an emergency hospital, where clinicians noted respiratory distress, weakness, tachypnea, tachycardia, and pyrexia (rectal temperature, $40.4^{\circ} \mathrm{C}$ ). Thoracic radiography revealed a severe alveolar pattern in the right caudal lung fields. Oxygen saturation was $70 \%$ while the dog was receiving supplemental oxygen. Blood work results were consistent with metabolic acidosis. The dog went into cardiopulmonary arrest that evening and could not be resuscitated. One of the dog's owners had become symptomatic for COVID-19 on July 6 and tested positive on July 13 .

Initial gross and histologic postmortem examination identified acute pneumonia, necrotizing rhinitis, mild hemothorax, mild to moderate right atrial and ventricular chamber dilatation, and chronic focal mural ulceration in the urinary bladder. Due to a COVID-19 positive household member, a respiratory panel and SARS-CoV-2 RT-PCR were run on nasal swabs collected postmortem and were negative for all targets except SARS-CoV-2 (Ct, 31 to 36). Formalinfixed, paraffin-embedded (FFPE) sections of lung and nasal turbinates were negative for SARS-CoV-2 by RTPCR and IHC at the CDC's IDPB. Escherichia coli was isolated by microbial culture from lung and spleen, and neutrophilic alveolar infiltrate was observed histologically. Gram-negative rods were seen within inflammation in the lungs, and IHC stains for $E$ coli were positive on lung tissue. These histopathology results were consistent with pneumonia without evidence of a viral component.
This dog had clinical signs consistent with SARSCoV-2 infection (Figure 1, 1.ii.), but the primary cause of death was attributed to bacterial bronchopneumonia (Figure 1, 2b.i.), and there was no evidence of virus in critical organs (Figure 1, 3a.i.). SARS-CoV-2 was determined to be an incidental finding based on the aforementioned findings and the duration of time between SARS-COV-2 infection and death (Supplementary Table S1).

\section{Case 6}

On September 24, 2020, a 5-year-old neutered male DSH cat with a recent history of upper respiratory signs developed neurologic abnormalities (head pressing and seizures) and weakness. The cat received 1 dose of clindamycin but became unresponsive and died at home on September 25. One person in the household had tested positive for SARS-CoV-2 on September 18.

Necropsy revealed nasal discharge, pulmonary congestion, and edema. Nasal and oral swabs were positive for SARS-CoV-2 (Ct, 25 to 29), and results of virus neutralization assay were positive (1:128). Fresh lung tissue was positive by RT-PCR (Ct, 37). FFPE lung and tracheal tissues were positive by RT-PCR for SARS-CoV-2 at the CDC's IDPB. All tissues were negative by SARS-CoV-2 IHC. Histopathological changes indicated suppurative meningoencephalitis, lymphoplasmacytic tracheitis, and mild myocardial disarray. In situ hybridization for SARS-CoV-2 was performed, revealing sparse staining in the tracheal submucosal glands and no staining in other tissues (lung, brain, spleen, liver, and muscle). Histopathological changes suggestive of a viral infection were not observed in lung tissue. Results of broad-range 16S rRNA gene PCR assays for bacterial detection in brain were indeterminate. Results of assays aimed at identifying another infectious cause of the meningoencephalitis were negative.

This cat had respiratory signs consistent with SARS-CoV-2 infection (Figure 1, 1.ii.), a significant comorbidity (suppurative meningoencephalitis; Figure 1, 2b.i.), and evidence of SARS-CoV-2 in lung tissue (Figure 1, 3a.ii.) but lacked associated histopathological lesions (Figure 1, 4a.i.). SARS-CoV-2 was considered the cause of tracheitis but incidental to the cause of death, which was attributed to suppurative meningoencephalitis (Supplementary Table S1).

\section{Case 7}

On October 2, 2020, a 16-year-old neutered male DSH cat presented to a veterinary hospital with increased respiratory effort beginning in late September. The cat was treated with cefovecin and methylprednisolone. Respiratory panel results were negative, and an oropharyngeal swab was positive for SARS-CoV-2 (Ct, 28). On October 4 , the owner reported that the cat had improved, but respiratory signs returned October 10 . On October 13, the cat presented to a veterinary hospital with respiratory distress and paradoxical breathing 
and was euthanized. Additional nasal, oropharyngeal, and rectal swabs, as well as heart, lung, and liver tissue samples were collected. The owner had tested positive for SARS-CoV-2 on September 18.

A postmortem oropharyngeal swab and frozen nasal passage and lung tissue were positive for SARSCoV-2 by RT-PCR (Ct, 30 to 36); heart and liver specimens were negative. Molecular evidence of SARSCoV-2 was not observed in 5 blocks of FFPE lung tissue evaluated at the CDC's IDPB. Histologically, moderate multifocal cardiomyocyte hypertrophy and disarray with degeneration, necrosis, and severe mineralization and fibrosis were seen in the heart. In the lungs, mild to moderate multifocal alveolar histiocytosis and interstitial fibrosis with edema, congestion, and hemorrhage were evident. Gross and histopathological findings in the heart were consistent with HCM, and lung lesions were consistent with heart failure. The cat had clinical signs consistent with SARS-CoV-2 infection (Figure 1, 1.ii.), a severe comorbidity (Figure $1,2 \mathrm{~b} . i$.$) , and presence of virus in critical tissues$ (Figure 1, 3a.ii.) but without associated histopathological lesions (Figure 1, 4a.i.). The cause of death was attributed to HCM with subsequent heart failure; SARS-CoV-2 was determined to be an incidental finding (Supplementary Table S1).

\section{Case 8}

On January 1, 2021, a 3-year-old neutered male DSH cat began vomiting and vocalizing in the litterbox. No respiratory signs were reported, and the cat was previously healthy. The owner did not take the cat for veterinary evaluation and elected to monitor overnight at home. The cat became weaker and died at home the next morning. The cat was brought to the veterinary hospital on January 2 for necropsy and cremation. Gross necropsy showed a urinary obstruction.

Two household members were symptomatic and recently confirmed positive for SARS-CoV-2. A nasal swab collected from the cat at necropsy was presumed positive for SARS-CoV-2 by RT-PCR (Ct, 29) on January 4 and confirmed positive via RT-PCR at the USDA NVSL (Ct, 30) on January 11. Lung tissue was RT-PCR negative for SARS-CoV-2 on January 11 at the NVSL.

SARS-CoV-2 infection was determined to be an incidental finding, as the cat showed no clinical signs consistent with SARS-CoV-2 infection (Figure 1, 1.i.), and necropsy findings suggested morbidity and death could be explained by urinary blockage (Figure 1, 2a.i.). Additionally, lung tissue was negative for SARSCoV-2 by RT-PCR.

\section{Case 9}

Beginning in March 2020, an 8-year-old neutered male shepherd mix dog developed increased respiratory rate and effort. These signs initially resolved without treatment but reappeared on approximately June 12. One owner became symptomatic on June 21 and tested positive for SARS-CoV-2 on a sample collected on June 24.
The dog was presented to a veterinarian on June 21. Thoracic radiographs showed a bronchointerstitial-to-alveolar pattern. The dog's condition later deteriorated, and the dog was presented to an emergency hospital with dyspnea and cyanosis on June 23. Microbial culture of a bronchoalveolar lavage sample was positive for Corynebacterium spp; CT showed chronic, diffuse pulmonary interstitial infiltrates with resultant cylindrical bronchiectasis; initial blood work was unremarkable (only mild neutrophilia noted); and bronchoscopy showed diffusely vascular and hyperemic airways and multiple small blood clots in small airways. Antimicrobials (ampicillin-sulbactam and enrofloxacin), corticosteroids, and supplemental oxygen were administered.

On June 26, veterinarians learned that the dog's owner had been diagnosed with COVID-19, and they pursued SARS-CoV-2 testing. Based on the clinical picture, diagnostics, imaging, bronchoalveolar lavage culture, and the lack of response to therapy, the clinicians suspected end-stage lung disease (interstitial fibrosis or a poorly exfoliative neoplasia) that had been exacerbated by an acute infection. The dog failed to improve with treatment and was euthanized on July 2 .

Results of respiratory PCR panel performed on the nasal swab collected on June 26 were negative for common causes of respiratory illness and positive for SARS-CoV-2 by RT-PCR (Ct, 21). Histologic examination of formalin-fixed lung tissues revealed severe, chronic lymphoplasmacytic bronchointerstitial pneumonia with marked interstitial fibrosis and bronchiolar squamous metaplasia consistent with chronic, severe lung disease. One section showed features of proliferative and organizing diffuse alveolar damage, with patchy type II pneumocyte hyperplasia and occasional fibroblastic proliferations; these finding suggested repair from a recent infection on the background of severe chronic lung disease. SARS-CoV-2 was detected in RNA extracted from FFPE tissue in 1 of 3 sections of lung by RT-PCR. However, viral antigen was not detected by IHC or in situ hybridization in the respiratory tissues.

This dog had clinical signs consistent with SARSCoV-2 infection (Figure 1, 1.ii), significant comorbidities (Figure 1, 2b.i.), presence of SARS-CoV-2 in critical tissues (Figure 1, 3a.ii.), and associated histopathological changes (Figure 1, 4a.ii.). Therefore, the final determination was that acute SARS-CoV-2 infection contributed to this animal's death, but the primary cause was chronic interstitial lung disease.

\section{Case 10}

On December 10, 2020, a 4-year-old neutered male domestic medium-hair cat became lethargic and inappetent. On December 13, the cat began to show signs of respiratory distress and was seen at 3 veterinary clinics. On December 15, the owner elected euthanasia due to the cat's rapid, progressive clinical deterioration. Both human household members tested positive for COVID-19 on December 5 and 21.

A necropsy was performed on the cat. Nasal, tracheal, oropharyngeal, and rectal swabs collected 
on December 18 were positive for SARS-CoV-2 by RT-PCR (Ct, 14 to 16). On histologic examination, bronchointerstitial pneumonia, acute myocardial degeneration and necrosis, and mild HCM were observed. ${ }^{21}$ A necropsy was performed. Nasal, tracheal, oropharyngeal, and rectal swabs collected on December 18 were positive for SARS-CoV-2 by RT-PCR (Ct, 14 to 16). On histologic examination, bronchointerstitial pneumonia, acute myocardial degeneration and necrosis, and mild HCM were observed. ${ }^{21}$ Live virus was isolated from nasal, tracheal, oropharyngeal, and rectal swabs, as well as heart and lung tissue. Both in situ hybridization and IHC results were positive. Feline respiratory panel results were positive for $M y$ coplasma felis.

This cat had clinical signs consistent with SARSCoV-2 (Figure 1, 1.ii.), subclinical comorbidities (mild HCM; Figure 1, 2b.ii.), evidence of SARS-CoV-2 in critical tissues (Figure 1, 3b.ii.), and histopathological changes attributed to SARS-CoV-2 in critical tissues (Figure 1, 4b.ii). ${ }^{21}$ Therefore, infection with SARS-CoV-2 was determined to be the primary reason for euthanasia.

\section{Discussion}

SARS-CoV-2 infections in companion animals are thought to result in mild illness or subclinical infection. In the US, from March 2020 to January 2021, 11\% (10/94) of confirmed SARS-CoV-2-positive companion animals died shortly before or after they tested positive. One-health investigations were conducted by public health and animal health officials, in consultation with attending veterinarians and pathologists, to characterize clinical illness and determine the role of SARS-CoV-2 in the clinical outcome of these 10 dogs and cats. An algorithm was developed to standardize investigations of these cases, determine the necessity of certain diagnostic procedures, and assign the role of SARS-CoV-2 in these deaths (Figure 1). Our designation using the algorithm reflects the most logical determination of the role that SARS-CoV-2 played in an animal's death, based on all available information obtained for each case. SARS-CoV-2 was determined to be a contributing factor in the death of $1 \mathrm{dog}$ and the primary cause of clinical signs that led to euthanasia for 1 cat, the first such report in companion animals with natural infection globally.

The CDC recommends testing for SARS-CoV-2 in companion animals with an epidemiological link to people with COVID-19 that show clinical signs consistent with SARS-CoV-2 infection. ${ }^{14}$ Four animals included in this case series did not fit this testing criteria; the animals had no signs consistent with SARS-CoV-2 infection. These animals had significant comorbidities that fully explained their clinical signs and death. Owners, veterinarians, and health officials should carefully consider the purpose of testing for SARS-CoV-2 in domestic animals, as there are currently no pathogen-specific therapies. These findings supported other research ${ }^{6-9}$ describing subclinical animal infections with SARS-CoV-2. The clinical relevance of a SARS-CoV-2 diagnosis in a critically ill animal should be cautiously interpreted.

Significant comorbidities observed in these cases include HCM, severe chronic lung disease, spinal neoplasia or intervertebral disk disease, hemic neoplasia, intracranial Schwannoma, bacterial bronchopneumonia, urinary obstruction, and suppurative meningoencephalitis. Despite most cases having severe comorbidities and epidemiological links to COVID-19-positive owners, SARS-CoV-2 was determined to play a role in only 2 deaths. The only animal that was concluded to have died as a result of SARS-CoV-2 infection (case 10) was only 4 years old and had no significant health conditions predisposing it to severe disease. In humans, the vast majority of deaths occur in persons with significant underlying health conditions, but people who are apparently healthy can succumb to infection. ${ }^{22}$ Hypertrophic cardiomyopathy was observed in several of the cats in the present case series. The significance of this correlation has yet to be established. However, in humans, upregulation of angiotensin-converting enzyme 2 receptors in cardiomyocytes has been observed in patients with HCM. This may provide evidence for a pathological relationship between SARS-CoV-2 infection and associated myocarditis. ${ }^{23}$ Improvements in surveillance for infections and thorough evaluation of cases with severe outcomes will improve our understanding of the range of health impacts in companion animals.

Full necropsies were conducted for 6 dogs and cats in the present study, and a partial necropsy was performed on a seventh. Determination of the role of acute infections like SARS-CoV-2 is difficult in animals with severe underlying health conditions without extensive postmortem evaluation and diagnostic testing. Necropsy and advanced detection methods necessary to characterize the virus's effects, such as in situ hybridization and IHC tests specific for SARS-CoV-2, require specialized equipment, facilities, and personnel. ${ }^{18}$ Veterinarians who suspect that SARS-CoV-2 may have contributed to severe illness in an animal, with consistent clinical signs and exposure, should immediately notify animal health and public health officials to facilitate further investigation. ${ }^{24,25}$ In some investigations, additional testing has been made possible through collaboration with and support from the US FDA's Veterinary Laboratory Investigation and Response Network (VetLIRN) laboratories. ${ }^{26}$

Of the 6 cases ( 2 dogs, 4 cats) in which tissues were evaluated histologically, 3 had viral lesions in respiratory and cardiac tissues, including the trachea, lungs, and heart. Rhinitis and tracheitis were each observed in 2 animals. It is unclear whether rhinitis was caused by SARS-CoV-2 or was preexisting and favored transmission. Although SARS-CoV-2 was found in lung tissue of 4 animals, it was not consistently found in all lung lobes tested, or positive results were not repeatable. This has been observed in humans and highlights the importance of adequate tissue sampling. ${ }^{18}$ Histopathological changes in the lung and up- 
per airway tissue seen in cases 6 and 7 had shared features observed in feline SARS-CoV-2 challenge studies. ${ }^{27}$ However, these changes were not consistently detected. Animals that fit the testing criteria should be thoroughly evaluated similarly to the process described here. In cases where all information has been collected, a simplified algorithm (Figure 2) helps to retrospectively evaluate the role of the virus in the course of disease.

This case series highlights several challenges of investigating and determining the role of natural SARS$\mathrm{CoV}-2$ infection in the clinical outcomes of companion animals, and the importance of assessing clinical signs in light of comorbidities, necropsy results, and histopathological and tissue staining results. Not all animals in this case series with severe outcomes had clinical signs consistent with SARS-CoV-2 infection. Most animals had significant comorbidities affecting clinical course, necropsy findings, and tissues evaluation. In animals with nonspecific clinical signs, necropsy, or histopathological changes, advanced diagnostic techniques helped identify changes attributable to virus presence in the tissue rather than detection due to vire- mia or cross-contamination. Limitations of diagnostic tests must be considered, as virus may be unevenly or sparsely distributed in tissue. Histopathological changes were not observed in all animals with natural infection, and severity varied, perhaps due to differences in dose, inoculation route, or genetic factors associated with species or purpose-bred laboratory animals evaluated in challenge studies. More research is needed to further evaluate and characterize the clinical and histologic manifestations of natural SARS-CoV-2 infection in companion animals.

\section{Acknowledgments}

The authors declare that there were no conflicts of interest.

The findings and conclusions in this report are those of the authors and do not necessarily represent the views of the CDC or USDA.

The authors would like to acknowledge members of CDC's COVID-19 One Health Working Group; Julu Bhatnagar, Lindsey Estetter, Marlene DeLeon-Carnes, Roosecelis Martines, Brigid Bollweg, and Luciana Flannery from IDPB's molecular and core pathology teams; and Drs. Megan Jacobs, Tatiane Watanabe, Robert B. Cohen, and Steve Brady and Julia Livengood, Roger Charles, and Nagaraja Thirumalapura for technical assistance, case referral, and follow-up.

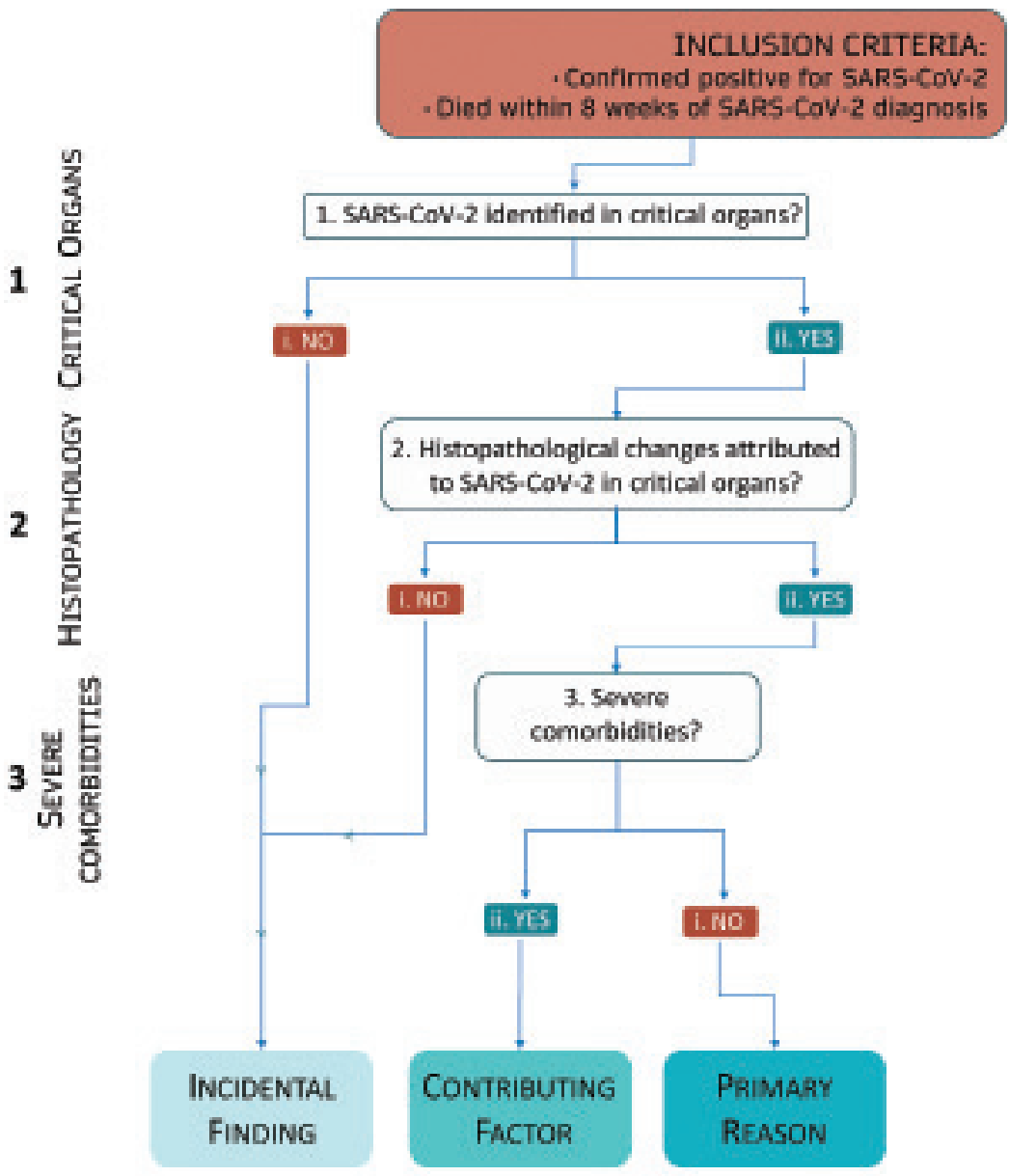

Role of SARS-CoV-2 in Companion Animal Death/Euthanasia

Figure 2-Algorithm used to determine the contribution of SARS-CoV-2 in the death of dogs and cats, among animals undergoing necropsy and histopathological evaluation.

\section{References}

1. Zhou P, Yang X-L, Wang X-G, et al. A pneumonia outbreak associated with a new coronavirus of probable bat origin. Nature. 2020;579(7798):270-273.

2. Andersen KG, Rambaut A, Lipkin WI, Holmes EC, Gary RF. The proximal origin of SARS-CoV-2. Nat Med. 2020;26(4):450-452.

3. Lau SKP, Luk HKH, Wong ACP, et al. Possible bat origin of severe acute respiratory syndrome coronavirus 2. Emerg Infect Dis. 2020;26(7):1542-1547.

4. Ghai RR, Carpenter A, Liew A, et al. Animal reservoirs and hosts for emerging alphacoronaviruses and betacoronaviruses. Emerg Infect Dis. 2021;27(4):1015-1022.

5. World Organisation for Animal Health. COVID-19 portal: events in animals. Accessed April 5, 2021. https://www.oie.int/en/scientificexpertise/specific-information-and-recommen dations/questions-and-answers-on-2019novelcoronavirus/events-in-animals/

6. Sit THC, Brackman CJ, Ip SM, et al. Infection of dogs with SARS-CoV-2. Nature. 2020;586(7831):776-778

7. Newman A, Smith D, Ghai RR, et al. First reported cases of SARS-CoV-2 infection in companion animals-New York, MarchApril 2020. MMWR Morb Mortal Wkly Rep. 2020;69(23):710-713.

8. Sailleau C, Dumarest M, Vanhomwegen J, et al. First detection and genome sequencing of SARS-CoV-2 in an infected cat in France. Transbound Emerg Dis. 2020;67(6):2324-2328.

9. Patterson EI, Elia G, Grassi A, et al. Evidence of exposure to SARS-CoV-2 in cats and dogs from households in Italy. Nat Commun. 2020;11(1):6231. doi:10.1038/ s41467-020-20097-0

10. Hosie MJ, Epifano I, Herder V, et al. Detection of SARS-CoV-2 in respiratory samples from cats in the UK associated with human-to-cat transmission. Vet Rec. 2021;188(8):e247. doi:10.1002/vetr.247 
11. Segalés J, Puig M, Rodon J, et al. Detection of SARS-CoV-2 in a cat owned by a COVID-19-affected patient in Spain. Proc Natl Acad Sci U S A. 2020;117(40):24790-24793.

12. USDA. Cases of SARS-CoV-2 in animals in the United States Accessed April 5, 2021. https://www.aphis.usda.gov/aphis/ ourfocus/animalhealth/sa_one_health/sars-cov-2-animals-us

13. USDA APHIS Veterinary Services. SARS-CoV-2. Case Definition - June 18, 2020. USDA; 2020.

14. CDC. Evaluation for SARS-CoV-2 testing in animals. Accessed April 5, 2021. https://www.cdc.gov/coronavirus/2019-ncov/ animals/animal-testing.html

15. USDA. FAQ for state animal and public health officials on animal coronavirus testing. Accessed April 5, 2021. https:// www.aphis.usda.gov/animal_health/one_health/downloads/faq-sapho-on-companion-animal-testing.pdf

16. USDA. SARS-CoV-2 virus in animals: testing strategies and reporting expectations. Accessed April 5, 2021. https://www. aphis.usda.gov/animal_health/one_health/downloads/sarscov2-testing-strategies.pdf

17. CDC. One health toolkit for health officials managing compan ion animals with SARS-CoV-2. Accessed April 5, 2021. https:// www.cdc.gov/coronavirus/2019-ncov/animals/toolkit.html

18. Martines RB, Ritter JM, Matkovic E, et al. Pathology and pathogenesis of SARS-CoV-2 associated with fatal coronavirus disease, United States. Emerg Infect Dis. 2020;26(9):2005-2015.

19. USDA. SARS-CoV-2. Case definition - June 11, 2021. Accessed April 5, 2021.https://www.aphis.usda.gov/animal_health/ one_health/downloads/SARS-CoV-2-case-definition.pdf

20. Lu X, Wang L, Sakthivel S, et al. US CDC real-time reverse transcription PCR panel for detection of severe acute respiratory syndrome coronavirus 2. Emerg Infect Dis. 2020;26(8):1654-1665.
21. Carvallo FR, Martins M, Joshi LR, et al. Severe SARS-CoV-2 infection in a cat with hypertrophic cardiomyopathy. Viruses 2021;13:1510.

22. Cunningham JW, Vaduganathan M, Claggett BL, et al. Clinical outcomes in young US adults hospitalized with COVID-19. JAMA Intern Med. 2020;181(3)379-381.

23. Tucker NR, Chaffin M, Bedi KC Jr, et al. Myocyte-specific upregulation of ACE2 in cardiovascular disease. Circulation. 2020;142(7):708-710.

24. CDC. What to do if your pet tests positive for the virus that causes COVID-19. Accessed April 5, 2021. https://www.cdc. gov/coronavirus/2019-ncov/daily-life-coping/positive-pet. html

25. CDC. Interim infection prevention and control guidance for veterinary clinics treating companion animals during the COVID-19 response. Accessed April 5, 2021. https://www.cdc.gov/ coronavirus/2019-ncov/community/veterinarians.html

26. FDA. Vet-LIRN SARS-CoV-2 supplemental necropsy sample inventory checklist. Accessed April 5, 2021. https://www. fda.gov/animal-veterinary/science-research/vet-lirn-sarscov-2-supplemental-necropsy-sample-inventory-checklist

27. Bosco-Lauth AM, Hartwig AE, Porter SM, et al. Experimental infection of domestic dogs and cats with SARS-CoV-2: pathogenesis, transmission, and response to reexposure in cats. Proc Natl Acad Sc US A. 2020;117(42):2638226388.

\section{Supplementary Materials}

Supplementary materials are posted online at the journal website: avmajournals.avma.org 